Fi el d- Effect Transi st or wi th Deposi ted Graphi te Thi n Film

\begin{tabular}{|l|l|}
\hline 著者 & $\begin{array}{l}\text { I nokawa Hi r oshi, Nagase Nasao, H r ono Shi ger u, } \\
\text { Got o Toui chi ro, Yamaguchi H r oshi, Tor i m t su } \\
\text { Kei i chi }\end{array}$ \\
\hline $\begin{array}{l}\text { j our nal or } \\
\text { publ i cat i on t i tl e }\end{array}$ & Japanese Jour nal of Appl i ed Physi cs \\
\hline vol une & 46 \\
\hline nunber & 4B \\
\hline page range & $2615-2617$ \\
\hline year & $2007-04$ 24 \\
\hline 出版者 & Japan Soci et y of Appl i ed Physi cs \\
\hline 権利 & $\begin{array}{l}\text { Copyr i ght ( C) 2007 The Japan Soci et y of } \\
\text { Appl i ed Physi cs }\end{array}$ \\
\hline URL & ht t p: //hdl . handl e. net /10297/9989 \\
\hline
\end{tabular}




\title{
Field-Effect Transistor with Deposited Graphite Thin Film
}

\author{
Hiroshi Inokawa*, Masao Nagase ${ }^{1}$, Shigeru Hirono ${ }^{2}$, Touichiro Goto ${ }^{1}$, \\ Hiroshi Yamaguchi ${ }^{1}$, and Keiichi Torimitsu ${ }^{1}$
}

Research Institute of Electronics, Shizuoka University, 3-5-1 Johoku, Naka-ku, Hamamatsu 432-8011, Japan

${ }^{1}$ NTT Basic Research Laboratories, NTT Corporation, Atsugi, Kanagawa 243-0198, Japan ${ }^{2}$ NTT Afty Engineering Corporation, Hachioji, Tokyo 192-0918, Japan

By using a bottom-gate top-contact field-effect transistor structure, the field effect of graphite-rich carbon nanocrystallite thin films deposited by electron cyclotron resonance sputtering was investigated. An appreciable ambipolar field effect was observed at the film edge where the thickness was vanishing. On-off current ratios of 2 and 7 were attained at 294 and $150 \mathrm{~K}$, respectively.

KEYWORDS: graphite, field-effect transistor (FET), electron cyclotron resonance (ECR) sputtering

*E-mail address: inokawa06@rie.shizuoka.ac.jp 


\section{Introduction}

Recent observations have revealed that graphite, particularly single- or few-layer graphite, is very attractive as an electronic material due to the extremely small effective masses and high mobilities of its electrons and holes ${ }^{1-4)}$. Among the carbon-related materials, carbon nanotubes have also drawn attention due to their excellent transport properties ${ }^{5)}$, but the control of the large number of geometrical parameters, i.e., position, direction, length, diameter, and chirality, is a difficult task. In this sense, graphite, as a two-dimensional sheet, is more suited for electron devices because of the smaller number of geometrical parameters that must be controlled. However, the handling techniques for few-layer graphite are still in its infancy, and there is no clear prospect for extensive applications. Considering these points, it is worthwhile to research the possibilities of deposited graphite thin films. Although such materials are usually polycrystalline and may not behave ideally, even a fraction of the intrinsic property is still valuable. Actually, Nagase et al. recently reported the pseudo-field-effect transistor (FET) using a carbon film deposited by electron cyclotron resonance (ECR) sputtering despite the high parasitic series resistance of the probes and/or contacts ${ }^{6)}$. Here, by using a thin-film FET structure with low parasitic resistance, the characteristics of the ECR-sputtered carbon film are investigated in detail.

\section{Device Fabrication}

FETs were fabricated using shadow mask depositions of carbon $(50 \mathrm{~nm})$ and metal $(100 \mathrm{~nm}$ $\mathrm{Au} / 10 \mathrm{~nm} \mathrm{Ti}$ ) from different incident angles. Figure 1(a) shows the plane view of the deposition mask and the deposited metal/carbon films. The sizes of the suspended mask $(L, W)$ were varied to obtain various channel lengths and widths of the FET. Figure 1(b) shows the cross-sectional view of the FET. The substrate was used as the gate, and the deposited metal was used as the source/drain 
electrodes (bottom-gate, top-contact structure). The gate oxide thickness was $20 \mathrm{~nm}$.

Note that this fabrication method is extremely useful in efficiently characterizing materials for FETs, because it involves only a two-step process of depositing the channel and electrode materials to complete the FET without additional lithographic and etching processes. Sensitive materials that cannot endure the complex processes can also be handled without degradation.

The carbon films were deposited by ECR plasma sputtering at room temperature under an $\mathrm{Ar}$ pressure of $5 \times 10^{-2} \mathrm{~Pa}$. A graphite target was sputtered with a DC bias of $-500 \mathrm{~V}$, and the sputtered carbon was deposited on the substrate at a rate of $4.5 \mathrm{~nm} / \mathrm{min}$. During the deposition, the film surface was simultaneously irradiated with low-energy ions with an acceleration voltage of approximately $20 \mathrm{~V}$.

It is reported that this kind of film consists of 2 5-nm-scale crystallites of graphite, and the content of the graphitic carbon can be evaluated by X-ray photoelectron spectroscopy (XPS) ${ }^{7}$. We reconfirmed the characteristics of the film by XPS spectrum analysis, as shown in Fig. 2. The C $1 s$ spectrum can be decomposed into four peaks. The first two peaks at lower energies are assigned to $\mathrm{sp}^{2}(\mathrm{C}=\mathrm{C})$ and $\mathrm{sp}^{3}(\mathrm{C}-\mathrm{C})$ bonds, respectively, and their intensity ratio is 1:0.28, indicating that the film is rich in graphite ${ }^{8)}$. The other small peaks at higher energies are assigned to the C-O bond that originated from the adsorbed oxygen ${ }^{7}$. The sheet resistance of the 50 -nm-thick film was measured, using a four-point probe, to be $2.7 \mathrm{k} \Omega$, corresponding to a resistivity of $14 \mathrm{~m} \Omega \cdot \mathrm{cm}$. This is one to two orders of magnitude higher than that of graphite parallel to the $c$ plane, but is far less than that of diamond, also supporting the assumption that the film is rich in graphite.

The $\mathrm{Ti}$ and Au films were deposited at room temperature by electron-beam evaporation under normal high vacuum $\left(\sim 10^{-4} \mathrm{~Pa}\right)$. The substrate was placed $90 \mathrm{~cm}$ away from the evaporation source, 
in order to minimize the half-shadow blur at the film edge.

\section{Electrical Characterization}

Devices with different $L$ and $W$ values were evaluated to find out the conditions needed to observe the field effect. Drain current $I_{\mathrm{D}}$ was measured with zero source voltage $V_{\mathrm{s}}$, drain voltages $V_{\mathrm{D}}$ of up to $2 \mathrm{~V}$, and gate voltages $V_{\mathrm{G}}$ from -6 to $6 \mathrm{~V}$. These $V_{\mathrm{G}}$ values roughly corresponded to the vertical electric field of $\pm 3 \mathrm{MV} / \mathrm{cm}$, which induces carriers in the channel. Since most of the device did not show the field effect, we will first show the conductance (or resistance) behaviors at a fixed $V_{\mathrm{G}}$.

Figure 3 shows the width dependence of the source-drain conductance measured at $V_{\mathrm{G}}=0$ and $V_{\mathrm{D}}=10 \mathrm{mV}$. For positive $W$, the conductance is proportional to $W$ as expected. From the slope of the fitted line and the sheet resistance obtained using a four-point probe, the parasitic series resistance is calculated to be $480 \Omega \cdot \mu \mathrm{m}^{9}$ ). This is comparable to those of metal-oxide-semiconductor field-effect transistors (MOSFETs) ${ }^{10)}$, indicating that the contact resistance is marginal. The temperature dependence of the conductance (inset) cannot be explained by simple thermally activated process. The hopping process might be a possibility ${ }^{11)}$, but the extracted exponent $\alpha$ is about 0.1 , which is far less than those for the variable-range hopping, i.e., $1 / 3$ for two-dimensional and 1/4 for three-dimensional conductions. A more precise evaluation with four-terminal measurement and/or in a wider temperature range is necessary.

Figure 4 is a logarithmic plot of the same conductance as in Fig. 3, showing the tail part of the conductance profile. The fitted curve is drawn on the assumption that the thickness distribution at the film edge is Gaussian with a standard deviation of $65 \mathrm{~nm}$ (inset) and the conductance is proportional to the thickness. At the thin part of the thickness distribution, where the conductance is 
less than $10^{-8} \mathrm{~S}$, modulation of the conductance by the gate electric field (field effect) was observed by separate measurements with varied gate voltages, i.e., $I_{\mathrm{D}}-V_{\mathrm{G}}$ measurements with $V_{\mathrm{D}}=10 \mathrm{mV}$ and $V_{\mathrm{G}}$ from -6 to $6 \mathrm{~V}$. The nominal thickness of the film corresponding to the field-sensitive device is calculated to be more than an order of magnitude smaller than that of a single graphite layer, i.e., $0.4 \mathrm{~nm}$. This is probably due to the failure of the assumption that the conductance of the film is proportional to its thickness. The accurate determination of the local film thickness or conducting an experiment with much smaller amounts of film deposition can be a future task.

Figure 5 shows the length dependence of the source-drain resistance. Since the channel area is shadowed from the carbon deposition when $L$ is larger than the height of the mask $(\sim 0.5 \mu \mathrm{m})$, the resistance increases rapidly. The curve for a larger $L$ is also fitted under the assumptions that the channel resistance is inversely proportional to the film thickness and the Gaussian thickness distribution has the same standard deviation of $65 \mathrm{~nm}$. The devices with a resistance higher than $10^{9} \Omega$ exhibited the field effect. Considering this and the results in Fig. 4 , we can estimate that the film edge with a vanishing thickness (much less than the deposited thickness of $50 \mathrm{~nm}$ ) plays an important role in the field effect, although the assumption for the fitting includes some ambiguity and the real thickness of the field-sensitive channel is not precisely determined at this moment.

Figure 6 shows the drain current-gate voltage characteristics for $L=0.75 \mu \mathrm{m}$ and $W=1.0 \mu \mathrm{m}$. The current increases on both the positive and negative sides of the gate voltage (ambipolar characteristics), presumably reflecting the semimetallic nature of graphite. The on-off current ratio tends to be larger for a lower drain voltage and a lower temperature. For $V_{\mathrm{D}}=0.5 \mathrm{~V}$, the on-off ratios of 2 and 7 were obtained at 294 and $150 \mathrm{~K}$, respectively. The expected on-off ratio at room temperature is $20 \sim 30$, if we assume that the intrinsic carrier concentration is $10^{-4}$ per carbon atom, and the maximum concentration induced by the electric field is $10^{13} \mathrm{~cm}^{-2}$. There is still room for 
improvement.

Apart from the on-off ratio, the drain current is appreciably small. Although the conduction mechanism is not clear as discussed in conjunction with Fig. 3, at least we understand that the hopping mechanism is not desirable. The small crystalline size of graphite and the $c$ plane being vertically oriented with respect to the film surface ${ }^{7)}$ might be the causes of the deviation from ideal conduction, i.e., band conduction of free carriers. Further improvement of the film quality in terms of such factors as crystalline size and orientation is necessary.

\section{Conclusions}

We investigated the field effect of graphite-rich carbon nanocrystallite thin films deposited by ECR sputtering, using a bottom-gate top-contact FET structure. An appreciable ambipolar field effect was observed at the film edge where the thickness was vanishing. On-off current ratios of 2 and 7 were attained at 294 and $150 \mathrm{~K}$, respectively. The results are encouraging further research on deposited graphite thin films for electronics applications.

\section{Acknowledgements}

We would like to express sincere thanks to Dr. Yukinori Ono and Dr. Satoru Suzuki for valuable discussions.

\section{References}

1) K. S. Novoselov, A. K. Geim, S. V. Morozov, D. Jiang, Y. Zhang, S. V. Dubonos, I. V. Grigorieva, and A. A. Firsov: Science 306 (2004) 666.

2) Y. Zhang, J. P. Small, W. V. Pontius, and P. Kim: Appl. Phys. Lett. 86 (2005) 
073104.

3) K. S. Novoselov, A. K. Geim, S. V. Morozov, D. Jiang, M. I. Katsnelson, I. V. Grigorieva, S. V. Dubonos, and A. A. Firsov: Nature 438 (2005) 197.

4) Y. Zhang, Y.-W. Tan, H. L. Stormer, and P. Kim: Nature 438 (2005) 201.

5) P. Avouris, J. Appenzeller, R. Martel, and S. J. Wind: Proc. IEEE 91 (2003) 1772.

6) M. Nagase, K. Nakamatsu, S. Matsui, H. Namatsu, and H. Yamaguchi: Jpn. J. Appl. Phys. 45 (2006) 2009.

7) S. Hirono, S. Umemura, M. Tomita, and R. Kaneko: Appl. Phys. Lett. 80 (2002) 425.

8) Although the G- and D-band signals in the Raman spectrum are more direct indicators of the presence and integrity of a graphite lattice, we chose XPS considering its sensitivity to thin samples. Evaluations using an advanced Raman spectrometer remain to be performed.

9) This series resistance is the sum of those on both the source and drain sides.

10) K. K. Ng and W. T. Lynch: IEEE Trans. Electron Devices 34 (1987) 503.

11) K. Matsuda, S. Hirono, and H. Takayanagi: Physica B 329-333 (2003) 1529. 


\section{Captions}

Fig. 1(a) Plane view of the deposition mask and deposited metal/carbon films, and (b) cross-sectional view of the FET. A two-step process of vertical deposition of carbon and oblique $\left(45^{\circ}\right)$ deposition of metal is used to complete the FET without additional lithographic processes.

Fig. $2 \mathrm{C} 1 \mathrm{~s}$ XPS spectrum of the deposited carbon film. The $\mathrm{sp}^{2}$ (graphite) component is predominant in the film. The small two peaks at higher energies are assigned to the C-O bond that originated from the adsorbed oxygen ${ }^{7}$.

Fig. 3 Width dependence of the source-drain conductance of the devices with $V_{\mathrm{G}}=0$ and $V_{\mathrm{D}}=10 \mathrm{mV}$. The slope of the fitted line is $7.8 \times 10^{-4} \mathrm{~S} / \mu \mathrm{m}$. Inset: Temperature dependence of the conductance.

Fig. 4 Logarithmic plot of the same conductance as in Fig. 3, showing the feature of the tail part of the thickness distribution at the film edge. From the separate evaluation similar to that in Fig. 6, the devices with a smaller conductance were found to be field-sensitive. Inset: Estimated thickness profile at the film edge.

Fig. 5 Length dependence of the source-drain resistance of the devices with $V_{\mathrm{G}}=0$ and $V_{\mathrm{D}}=10 \mathrm{mV}$. The resistance increases rapidly when the channel region is shadowed by an enlarged $L$ ( $>0.5 \mu \mathrm{m})$. The devices with a high resistance were found to be field-sensitive.

Fig. 6 Drain current-gate voltage characteristics for $L=0.75 \mu \mathrm{m}$ and $W=1.0 \mu \mathrm{m}$ at (a) $294 \mathrm{~K}$ and (b) $150 \mathrm{~K}$. On-off current ratios of 2 and 7 are attained, respectively. 
Fig. 1
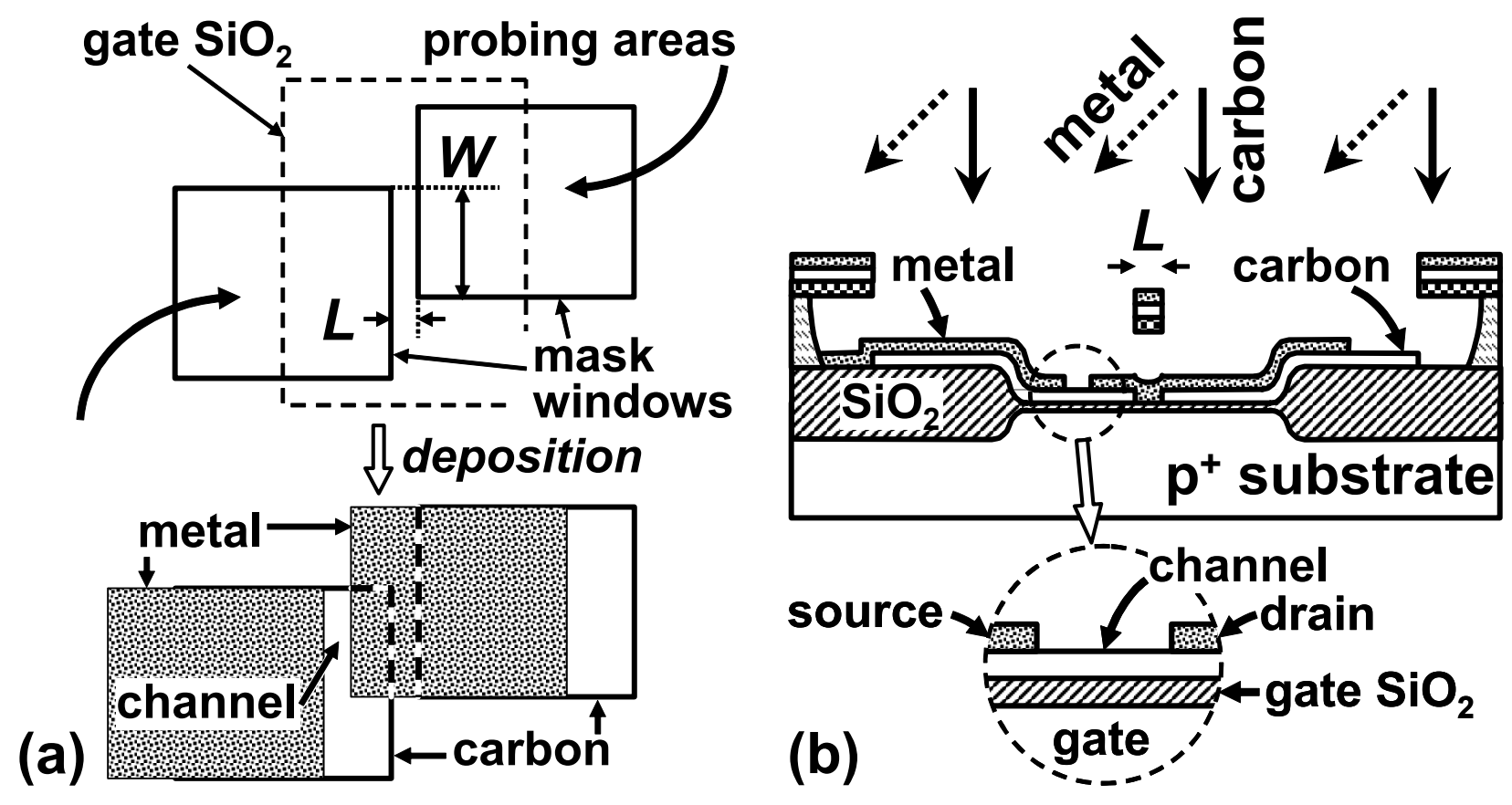

H. Inokawa, et al. 
Fig. 2

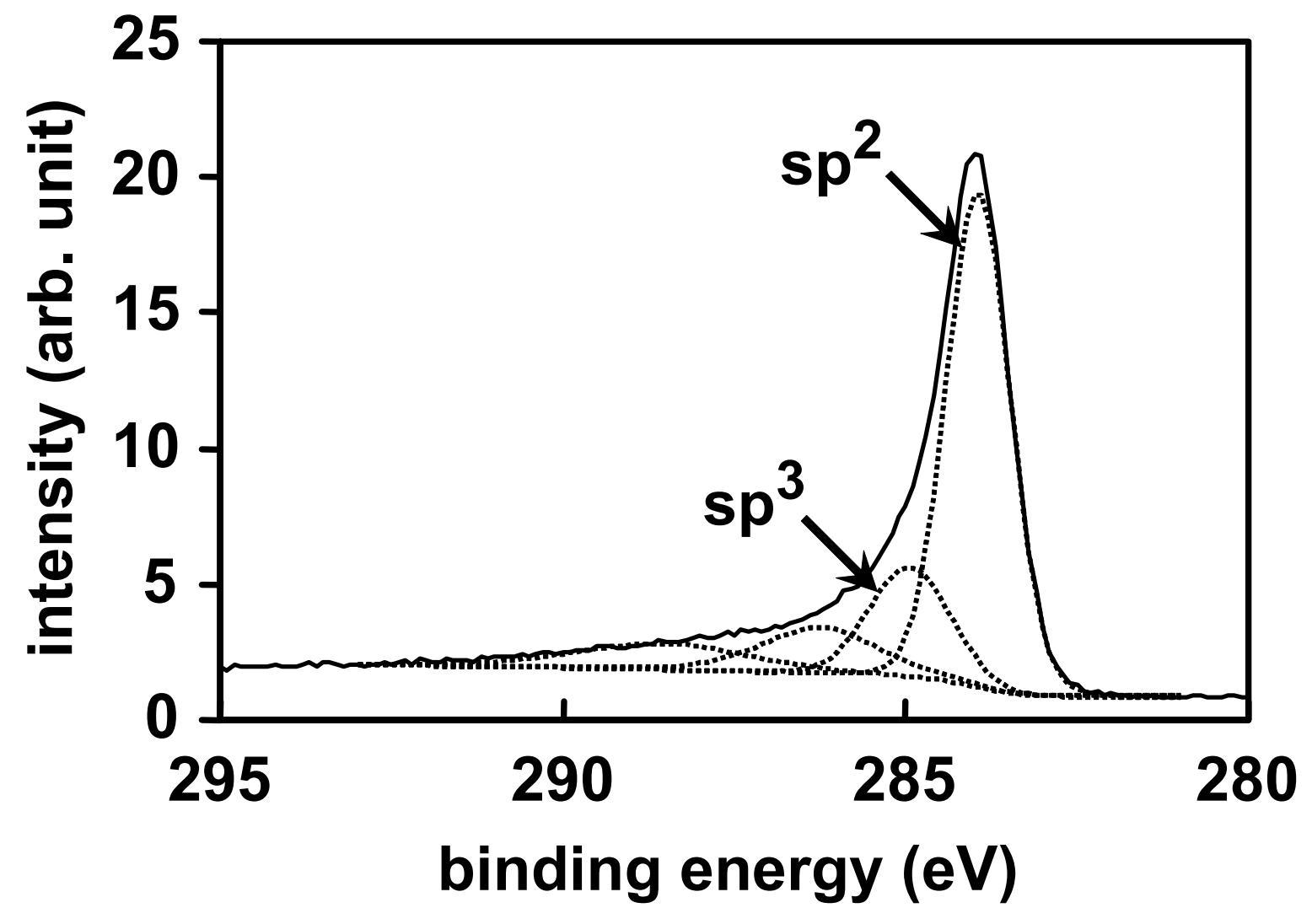

H. Inokawa, et al. 
Fig. 3

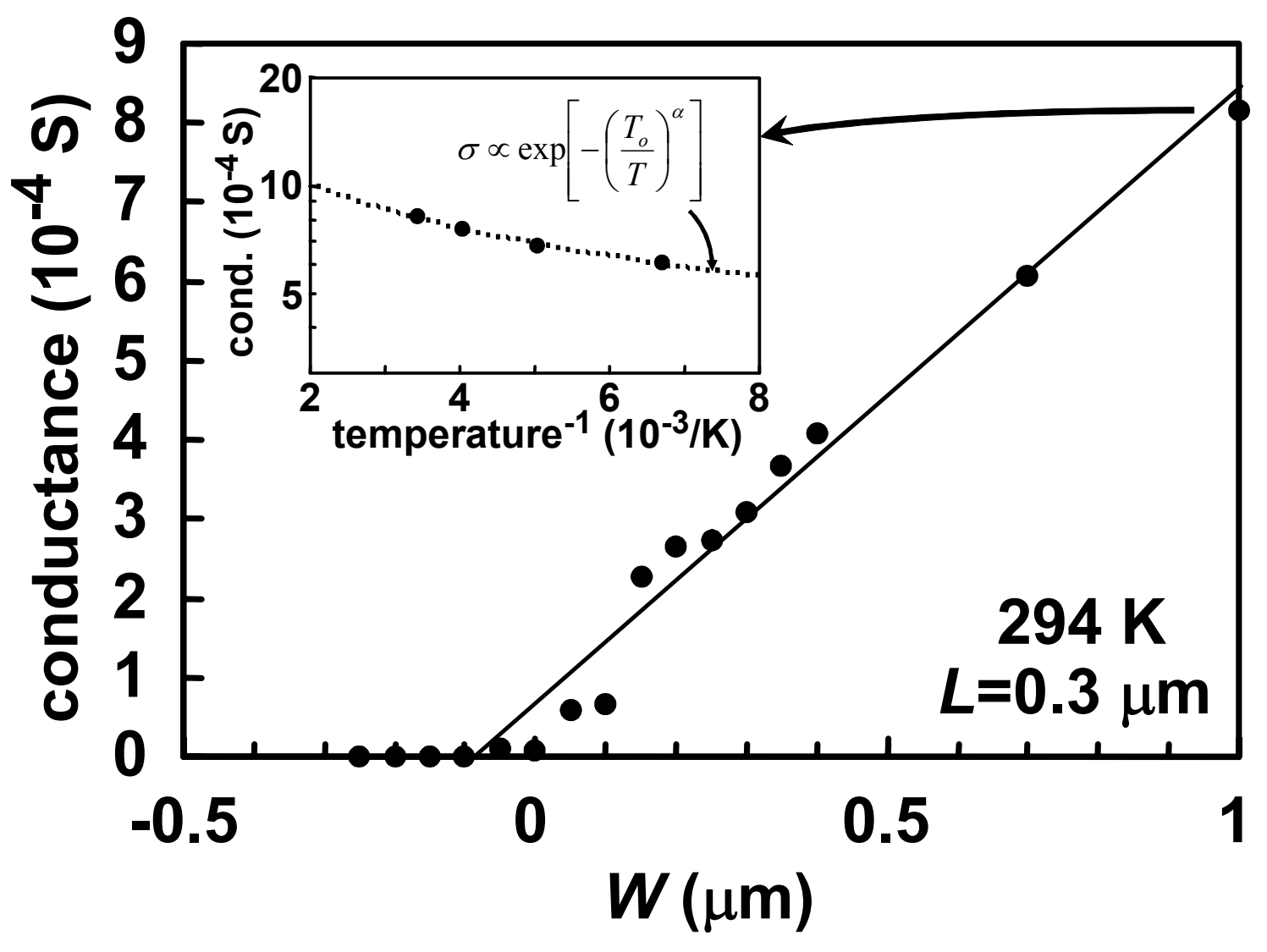

H. Inokawa, et al. 
Fig. 4

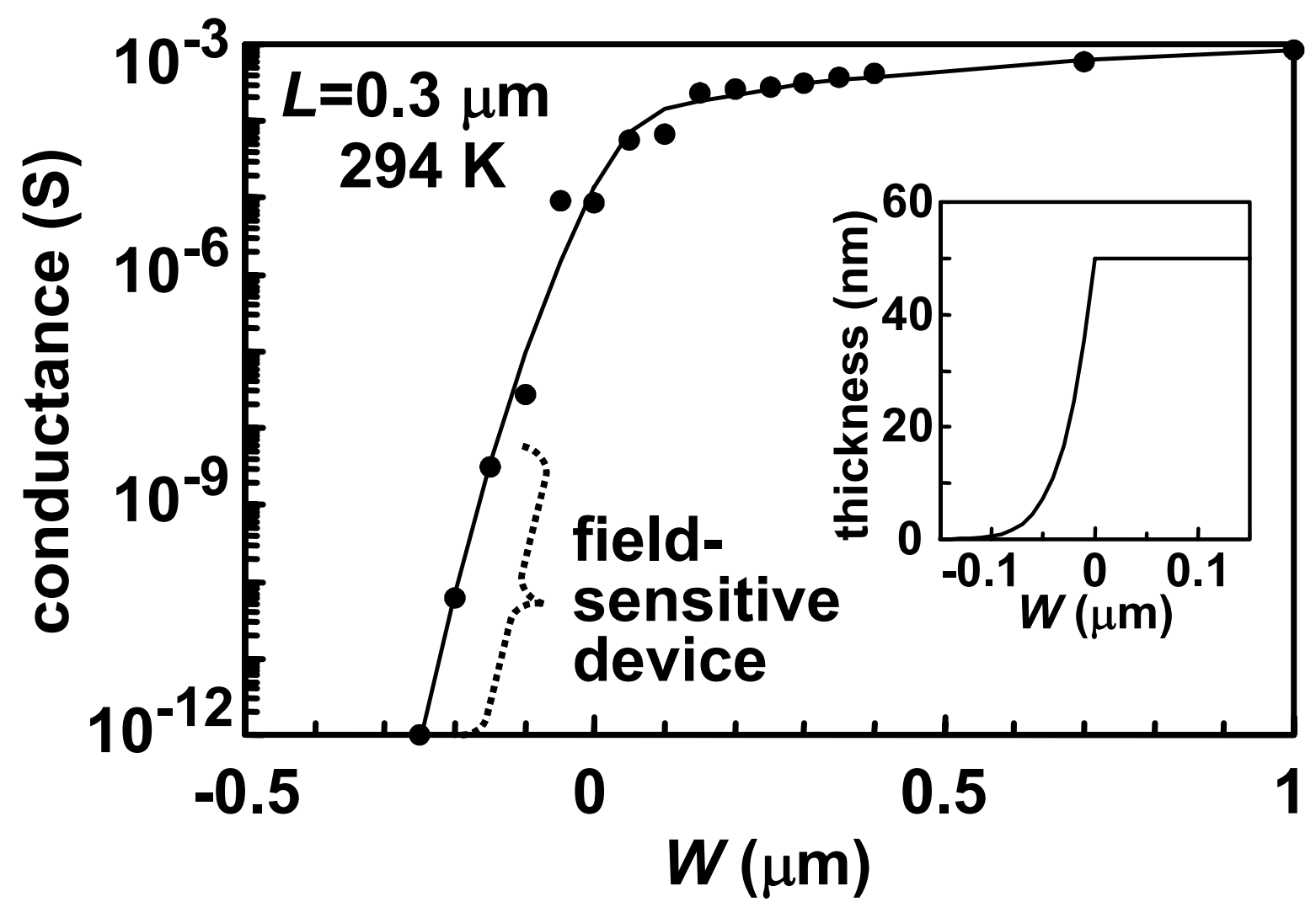

H. Inokawa, et al. 
Fig. 5

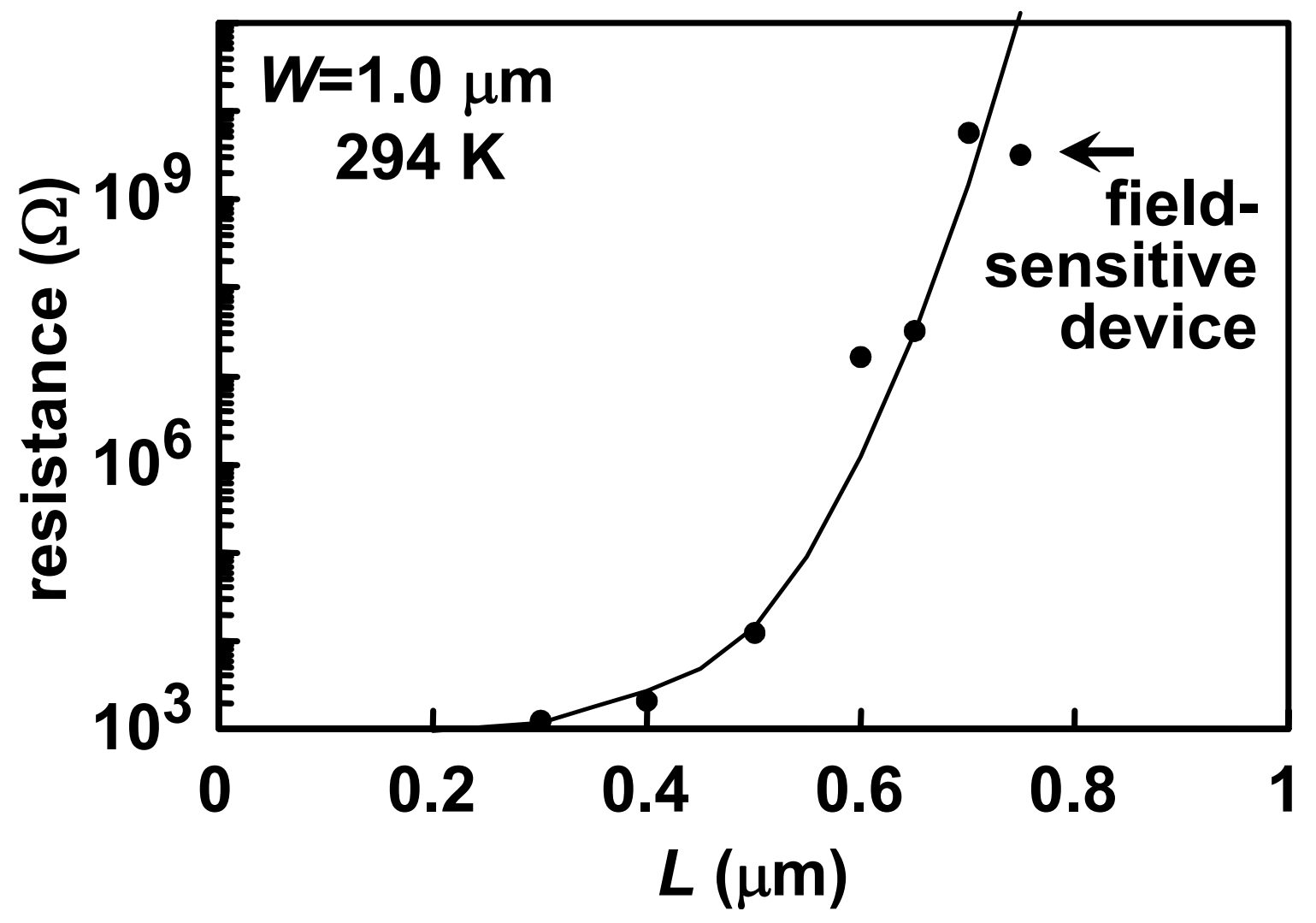

H. Inokawa, et al. 
Fig. 6
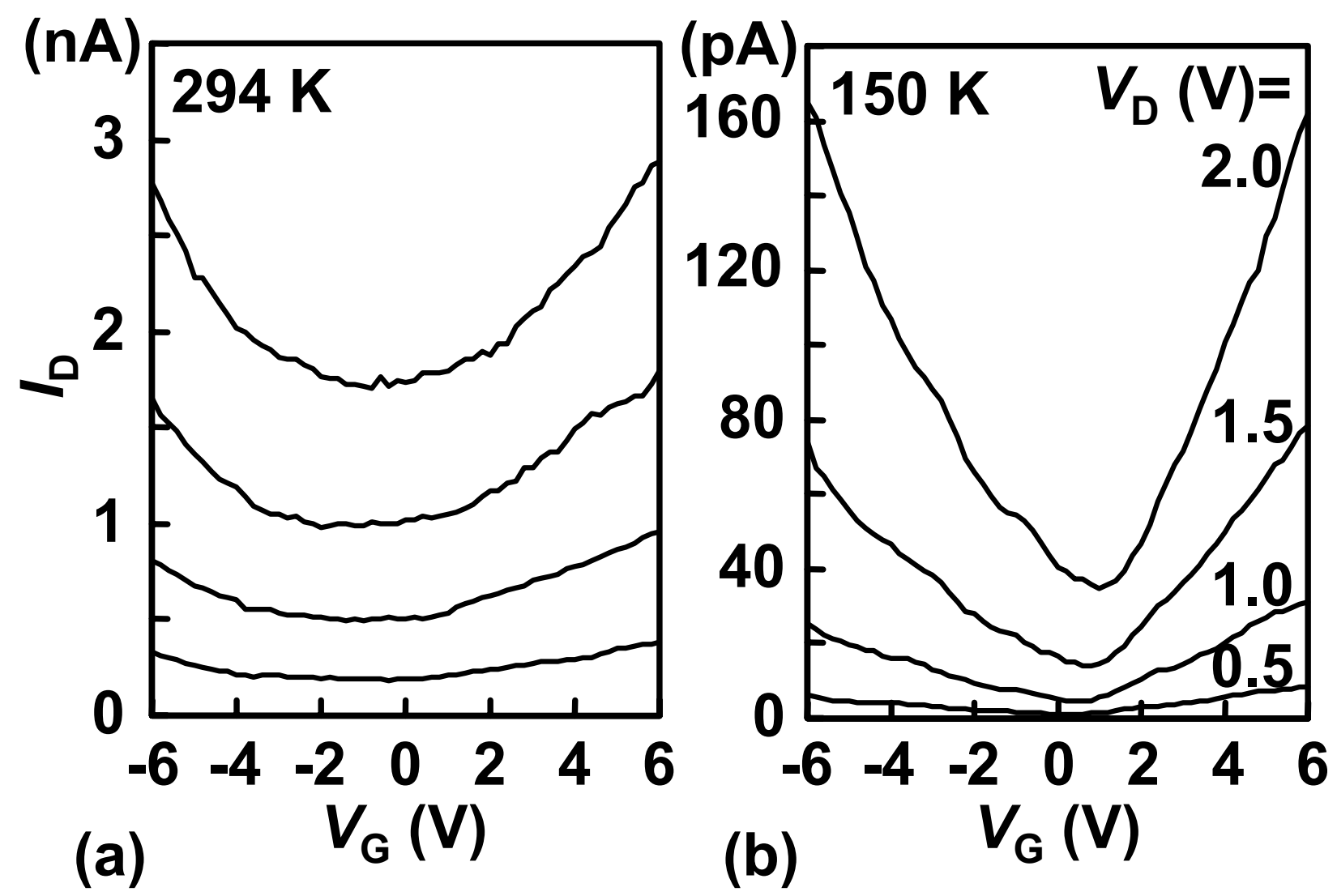

H. Inokawa, et al. 